Relations industrielles

Industrial Relations

\title{
The Management of Personnel Relations, History and Origins, Cyril C. Ling. Richard D. Irwin, Inc., Homewood, Illinois. 1965. 554 pages.
}

\section{Ronald Pleau}

\section{Volume 21, numéro 2, 1966}

URI : https://id.erudit.org/iderudit/027690ar

DOI : https://doi.org/10.7202/027690ar

Aller au sommaire du numéro

Éditeur(s)

Département des relations industrielles de l'Université Laval

ISSN

0034-379X (imprimé)

1703-8138 (numérique)

Découvrir la revue

Citer ce compte rendu

Pleau, R. (1966). Compte rendu de [The Management of Personnel Relations, History and Origins, Cyril C. Ling. Richard D. Irwin, Inc., Homewood, Illinois. 1965. 554 pages.] Relations industrielles / Industrial Relations, 21(2), 305-306. https://doi.org/10.7202/027690ar

Tous droits réservés @ C Département des relations industrielles de l'Université Laval, 1966
Ce document est protégé par la loi sur le droit d'auteur. L’utilisation des services d’Érudit (y compris la reproduction) est assujettie à sa politique d'utilisation que vous pouvez consulter en ligne.

https://apropos.erudit.org/fr/usagers/politique-dutilisation/ 
pour enfin peindre le système qui suivit la deuxième grande guerre.

Dans un système de relations industrielles comme celui qui prévaut dans I'industrie lithographique, la négociation des contrats collectifs de travail semble être affectée par l'individualisme des employeurs et des syndicats locaux, malgré l'effort pour négocier à l'échelle nationale. $\mathrm{Ce}$ niveau amène donc assez souvent des négociations informelles exigées par le climat de désaccord qui peut exister au moment du renouvellement d'une convention.

Depuis 1945, les travailleurs de l'industrie lithographique ont obtenu des conditions plus que favorables: semaine de travail de 35 heures pour la majorité d'entre eux, plans d'assurance-santé, d'assurance-vie et de pension. De plus, il semble qu'ils aient progressé plus ropidement sur le terrain des salaires que la plupart des travailleurs dans les autres métiers de l'imprimerie. Et comme conséquence de la négociation dans cette industrie, on a vu apparaittre un contrôle de l'offre de travail par les syndicats, contrôle qui s'est concrétisé d'une part par des restrictions sur le nombre de travailleurs et d'autre part par des restrictions sur les heures de travail. Le premier type de contrôle a donc amené une restriction de I'offre alors que le second servait d̀ créer une demande additionnelle. L'auteur termine son exposé en résumant la situation actuelle et en esquissant les tendances qui se dessinent pour l'avenir.

\section{Mathieu Vaillancourt}

The Management of Personnel Relations, History and Origins, Cyril C. Ling, Richard D. Irwin, Inc., Homewood, Illinois. 1965. 554 pages.

Comme l'indique bien le titre de ce volume, nous retrouvons dans ses pages I'histoire de la croissance de la fonction du personnel dans les firmes américaines.

Cet ouvrage volumineux est divisé en cinq grandes parties. Dans une première l'auteur introduit son sujet de façon à expliquer les différentes questions dont il traitera par la suite.

II débute son exposé par une description de l'évolution du contexte économique et social dans lequel a évolué la fonction du personnel après quoi il nous fournit un cadre de référence destiné à faciliter la définition et la compréhension des concepts employés dans cette étude. Puis il poursuit en nous donnant quelques origines de la pensée concernant le personnel pour terminer par une explication du plan de son volume avec les limitations et les avantages d'un tel travail.

Dans l'élaboration des faits historiques qui constituent la matière des quatre parties subséquentes, on remarque que l'ordre chronologique a été suivi. Ainsi le contenu se divise selon les périodes suivantes: de 1400 à 1890 (Partie II) i.e. le premier stage de développement, de 1890-1920 (Partie III) le second stage de développement, de 1920 à nos jours ou troisième stage de développement. Dans la partie $V$, on retrouve un bref sommaire de ce qui $s^{\prime}$ est fait dans le passé suivi d'un essai de projection pour savoir ce qui pourrait survenir dans l'avenir dans le domaine du personnel.

Dans la partie II donc, ce sont les influences de l'évolution industrielle et technologique, du mouvement du "Welfare », du mouvement syndical, de la législation dú travail, de la législation industrielle sur la fonction du personnel et des contributions à l'éducation de cette dernière qui font l'objet d'un examen plus approfondi. Ces sujets constituent autant de chapitres à l'intérieur desquels l'auteur passe en revue l'évolution industrielle de l'Europe et des Etats-Unis avec son impact sur la fonction du personnel (chap. 2), la définition, les objectifs, la patience, le travail dans la guilde et dans I'industrie moderne du mouvement du « Welfare » (chap. 3), les formes d'apprentissage, les débuts de l'éducation dans l'industrie et les facteurs politiques qui l'ont influencée (chap. 4), la croissance, les effets de la croissance des syndicats américains et de la législation ouvrière (chap. 5), l'évolution des lois concernant les salaires et les heures de travail, leurs effets sur la fonction du personnel, les lois de sécurité et d'accident du travail (chap. 6).

La section suivante est consacrée à la modernisation de la médecine industrielle, à ses origines, à son évolution, son statut, ses problèmes en relations avec le personne! (chap. 7), à la psychologie industrielle, ses origines, son développement et les contributions qu'elle a apportées à cette fonction de l'entreprise (chap. 8) aux contributions de la pensée du management i.e. du « planning " ou la prise de décision, à l'organisation, au leadership, à la motivation et au contrôle (chap. 9) à l'influence de la seconde guerre mondiale, et à ses effets sur les relations avec les employés (chap. 10), aux contributions internes telles les tendances vers la professionalisation (chap. 11). 
Puis en passant à la partie IV on retrouve les contributions cette fois de la sociologie industrielle et des découvertes et expériences dont elle a été le cadre (chap. 12), l'influence de la dépression économique (chap. 13) et de la seconde guerre (chap. 14) sur les différentes fonctions concernant la direction du personnel.

Somme toute ce volume pourra être de lecture intéressante et très utile d̀ tout étudiont, professeur ou professionnel intéressé à la direction du personnel, à la théorie de l'organisation, ò I'histoire des affaires, d̀ I'histoire économique ou à l'économique du travail.

\section{Ronald Pleau}

The Price Systems and Resource Allocation (3rd Edition), Richard $H$. Leftwich, Holt, Rinehart and Winston of Canada Ltd.

833 Oxford St., Toronto 18, Ont., 1966, 369 pages.

L'auteur expose comment le mécanisme des prix alloue les ressources rares de l'économie parmi ses besoins illimités. A cet égard, ce volume ne présente que peu de nouveau, si on le compare aux nombreux outres parus depuis plusieurs années sur le même sujet.

Nous sommes très heureux d'ajouter que ce volume se distingue cependant de plusieurs de ses semblables par la qualité de son exposé, clair, concis et complet. L'auteur fait preuve d'une excellente pédagogie. Et celle-ci sera d'autant plus appréciée des étudiants de l'économique que le contenu du volume se situe à un niveau, plutôt élémentaire de la micro-économique.

Signalons aussi l'intérêt du dernier chapitre qui représente la programmation linéaire. En effet, tout le monde sait que l'équilibre de la firme peut être déterminé par le marginalisme orthodoxe, d̀ la condition, toutefois, qu'il n'existe pas de limitations dans la quantité des facteurs de production utilisés. Or, de telles limitations constituent probablement la règle générale. C'est d'ailleurs ce chapitre qui constitue l'addition majeure de cette troisième édition.

Nous recommandons donc fortement cet excellent volume d̀ tous les intéressés d̀ ce sujet primordial en économique qu'est le mécanisme des prix et l'allocation des ressources.

Bertrand Belzile
Le Canada, dernière chance de l'Europe par Claude Julien. Grasset, Paris, 1965. 255 pages.

Cet ouvrage est d'abord écrit en fonction de lecteurs européens et porticulièrement des responsables de la politique en France.

L'auteur y développe la thèse suivante. Le destin de l'Europe est lié au sort du Canada. « Une fois dans son histoire la France a perdu le Canada au profit de l'Angleterre. De nos jours, si elles n'y prennent garde, la France et l'Angleterre vont perdre le Conada au profit des Etats-Unis. Et cette perte, pour l'Europe entière, serait infiniment plus grave que ne le fut pour la France la défoite de Montcalm. (53) Le Canada possède un énorme potentiel économique qui s'il venait à s'ajouter à la puissance des Etats-Unis interdirait tout équilibre entre les deux rives de I'Atlantique. Déjà le Canada est sous l'emprise des capitaux américains, mais la partie n'est pas perdue, car il y a encore place pour de vastes apports de capitaux, de techniciens et de travailleurs spécialisés.

Les lecteurs canadiens de langue française comme ceux de langue anglaise y trouveront aussi un appel au sens de la responsabilité à l'échelle mondiale. «Obnubilés par les difficiles relations entre le groupe onglophone et le groupe francophone, ils en viennent à oublier parfois leur problème numéro un, qu'ils connoissent pourtant bien, celui de leur dépendance éconornique, et donc politique, à l'égard des Etats-Unis. * (60). "Un Canada morcelé deviendrait pour l'emprise du capital américain une proie encore plus facile. L'indépendance du $\mathrm{Ca}$ nada, qui conditionne ainsi une certaine conception de l'Occident, dépend donc à la fois de l'Europe et des Canadiens euxmêmes. II est grand temps pour l'Europe de s'intéresser utilement au Canada en développant avec lui ses échanges commerciaux, en lui apportant des capitaux et des techniciens. II est grand temps, pour les Canadiens, d'envisager leurs propres problèmes dans une perspective non pas étroitement nationale, mais mondiale. w (61). On en vient ainsi à voir le rôle que le Québec est appelé à jouer sur l'avenir du monde, car c'est lui qui conteste les structures conadiennes mal adaptées aux nécessités présentes. C'est lui aussi qui peut jouer un rôle de moteur et qui est dे l'origine de solutions neuves.

Dans l'élaboration de sa thèse, l'auteur onolyse les différents éléments de la situotion économique, sociale et politique du 